standing, and lasts longer than six months. Pain is associated with dyspareunia and varicosities in lower limbs (LL) and genitals. It is challenging and important to consider atypical clinical presentations that simulate osteoarticular (OA) pathologies. It is usually diagnosed by Angio-CT, and a safe, definitive and successful treatment is the embolization of the affected vein.

Objectives: The objective of this study was to evaluate the characteristics of those patients diagnosed with PCS in our University hospital from January 2014 to May 2018, paying close attention to the atypical forms of presentation that simulate OA pathology.

Methods: We included all patients from our center who were operated by embolization due to a PCS from January 2014 to May 2018. Socio-demographic variables, forms of presentation, pain characteristics, associated symptoms, patient management and outcome data were collected.

Results: Sixty women were included with a mean age of 43 years at diagnosis, $87 \% \quad(n=52)$ were multiparous, with a mean of 2 previous pregnancies. In $95 \%$ (57) of all cases the duration of symptoms until the diagnosis exceeded 6 months. Patients were classified according to presence and location of pain in 4 groups: 1 . Women with AP pain, $23 \%$ $(14) ; 2$. Women with $\mathrm{OA}$ pain $5 \%(3) ; 3$. Women with mixed AP and $\mathrm{OA}$ pain, 59\% (35); and 4. Women with other symptoms $13 \%$ (8). Regarding patients from groups 2 and 3 (only OA pain or mixed pain) (38), $90 \%$ (34) of them presented low back pain, $53 \%$ (20) hip pain and $40 \%$ (15) sciatic pain. Only $5 \%$ (3) of all patients were evaluated by a rheumatologist.

As for the pain characteristics from groups 1, 2 and 3 (52), in $72 \%$ (37) of patients it was diurnal, in $48 \%$ (25) it worsened with menstruation, in $62 \%$ (32) it worsened with prolonged standing and in $35 \%$ (18) it worsened at rest. Among the associated manifestations, stand out the presence of LL varicose veins in $74 \%$ (38) of patients, genital varicosities in $58 \%$ (30), dyspareunia in $42 \%$ (22), dysmenorrhea in $40 \%$ (21), hemorrhoids in $37 \%$ (19) and dysuria in $18 \%$ (9). All patients underwent embolization of the affected vein, with an initial Visual Analogue Scale (VAS) mean of 7.38 over 10 , and final VAS mean of 2.63 . The mean recovery time was 36 days. The evolution was good or very good in $84 \%$ (32) of patients with mixed (AP and OA) pain and in $57 \%$ (8) of those who only had AP pain. Less than $2 \%$ (1) had a recurrence without the need for reoperation.

Conclusion: PCS is a rare entity, typically associated with long lasting AP pain, but, in more than half the cases, is accompanied with OA symptoms, mainly low back pain. It is important and challenging for the rheumatologist to identify these patients, since treatment is usually safe and effective, and diagnostic delay worsens their quality of life.

Disclosure of Interests: None declared

DOI: 10.1136/annrheumdis-2019-eular.5373

27. BACK PAIN, MECHANICAL MUSCULOSKELETAL PROBLEMS, LOCAL SOFT TISSUE DISORDERS

\section{THU0501 MUSCULOSKELETAL DISORDERS IN TYPE 1 AND 2 DIABETIC PATIENTS: PREVALENCE AND ASSOCIATION WITH MICROVASCULAR COMPLICATIONS OF DIABETE}

hamza toufik, Mohamed A.Ghassem, Julien Djossou, Najlae EL Ouardi, Abderrahim Majjad, Abdellah EL Maghraoui. Mohammed V Military Academic Hospital, Faculty of Medicine and Pharmacy, Mohammed V University, Rheumatology Department, Rabat, Morocco

Background: Diabetes mellitus (DM), a worldwide high prevalence dis ease, is associated with a large variety of musculoskeletal (MS) disorders. They are poorly treated, as compared to microvascular complications. However, they are a common source of disability.

Objectives: We designed this study to assess the prevalence of MS disorders among diabetic patients and their relation to microvascular complications of diabetes.

Methods: A cross-sectional study enrolled consecutive subjects with diabetes seen in the Endocrinology department. We recorded age of patients, sex, body mass index, type and duration of diabetes, Long-term glycemic control assessed by hemoglobin A1c levels, and lipid profile. Musculoskeletal and microvascular disorders assessment was done by detailed history with clinical examinations and investigations if needed.

Results: A total of 376 subjects were studied, $(84.6 \%$ had type 2 diabetes). The mean age was $52.5 \pm 13.9$ years, $41 \%$ had one or more microvascular complications, among which retinopathy was present in $28.2 \%$, nephropathy in $16.1 \%$ and neuropathy in $12.8 \%$. Moreover, $23.4 \%$ of the patients had one or more musculoskeletal disorders. Shoulder capsulitis was present in $12.5 \%$; carpal tunnel syndrome in $8.8 \%$; trigger finger in 5.9\%; and $2.9 \%$ had diabetic cheiroarthropathy. Dupuytren's contracture and Charcot foot, were found in $0.5 \%$ and $0.3 \%$ of the cases respectively. Symptomatic osteoarthritis was found in $19.4 \%$. Musculoskeletal disorders prevalence increased with age, diabetes duration, presence of dyslipidemia and various microvascular complications.

Abstract THU0460 -Table 1. Distribution of cases according to MS disorders in relation to type of diabetes

\begin{tabular}{|c|c|c|c|c|c|c|c|}
\hline \multirow[t]{3}{*}{ MS disorders } & \multicolumn{5}{|c|}{ Type of diabetes } & \multirow{2}{*}{\multicolumn{2}{|c|}{$\begin{array}{c}\text { Total } \\
\mathrm{N}=376\end{array}$}} \\
\hline & \multicolumn{2}{|c|}{ Type $1 \mathrm{~N}=58$} & \multicolumn{2}{|c|}{ Type $2 \mathrm{~N}=318$} & \multirow{2}{*}{$\begin{array}{l}\mathrm{P} \\
\%\end{array}$} & & \\
\hline & No. & $\%$ & No. & $\%$ & & No. & $\%$ \\
\hline Osteoarthritis & 2 & 3.4 & 71 & 22.3 & $0.001^{*}$ & 73 & 14.9 \\
\hline Shoulder capsulitis & 2 & 3.4 & 45 & 14.2 & $0.023^{*}$ & 47 & 12.5 \\
\hline Carpel Tunnel Syndrome & 5 & 8.6 & 28 & 8.8 & 0.969 & 33 & 8.8 \\
\hline Limited joint mobility & 0 & 0 & 11 & 3.5 & 0.151 & 11 & 2.9 \\
\hline Trigger Finger & 2 & 3.4 & 20 & 6.3 & 0.397 & 22 & 5.9 \\
\hline Dupuytren's contracture & 0 & 0 & 2 & 0.6 & 0.545 & 2 & 0.5 \\
\hline Charcot's Foot & 1 & 1.7 & 0 & 0 & 0.154 & 1 & 0.3 \\
\hline Total MS disorders & 10 & 17.2 & 78 & 24.5 & 0.149 & 88 & 23.4 \\
\hline
\end{tabular}

Conclusion: this study shows a high prevalence of musculoskeletal disorders in diabetic's patients which were significantly associated with advanced age, longer duration of diabetes, presence of dyslipidemia and microvascular complications.

Disclosure of Interests: : None declared

DOI: 10.1136/annrheumdis-2019-eular.6225

\section{THU0502 DIFFERENTIAL DIAGNOSIS OF MONOARTHRITIS: THREE CASES WITH PIGMENTED VILLONODULAR SYNOVITIS}

Serkan Turkucar, Ceyhun Acari, Hatice Adiguzel Dundar, Erbil Unsal. Dokuz Eylul University, Faculty of Medicine, Department of Pediatric Rheumatology, Izmir, Turkey

Background: Pigmented Villonodular Synovitis (PVNS) is a proliferative disorder of synovium that affects synovial joints, tendon sheaths and bursas. The estimated incidence is around 1.8 cases per million people in a population. PVNS is usually found in adults aged 20-50 years, but it may occur also in children. As it is a rare pathology in children, diagnosis is often delayed, and it is difficult to distinguish from Juvenile Idiopathic Arthritis (JIA), hemophilic arthropathy, tuberculosis, and other neoplastic processes.

Objectives: The aim of this case series is to emphasize PVNS in the differential diagnosis of monoarthritis, and the importance of interpretation of imaging, i.e. MRI.

Methods: Three pediatric PVNS cases who were misdiagnosed as monoarticular JIA and familial Mediterranean fever (FMF) are presented as case series.

Results: Case-1. A 14 year-old male had swelling and pain on his left knee for one year. An MRI of knee was reported as joint effusion in suprapatellar bursa, and he was referred to our clinic as monoarticular JIA. He was on methotrexate (MTX) $15 \mathrm{mg} / \mathrm{m}^{2} / \mathrm{wk}$. SC, and despite effective treatment there was no improvement. MRI was performed by a pediatric radiologist who was an expert on musculoskeletal diseases. Joint effusion and a lesion compatible with PVNS were observed in left suprapatellar recess (Figure-A). Arthroscopic synovectomy was performed and histopathological result was PVNS.

Case-2: A one year-old female had swelling on her right knee for three months after a minor trauma. On physical examination, she had effusion of the right knee which was confirmed on USG. She was diagnosed as monoarticular JIA and intraarticular triamcinolone hexacethonide injection was performed. She was unresponsive to adequate non-steroid drug therapy, and MTX was started. Despite intensive therapy, there was no change. MRI showed focal lesions with high signal in T2 weighted images in suprapatellar and intraarticular areas, and findings were evaluated as compatible with PVNS (Figure-B). Total synovectomy was performed and PVNS was confirmed in pathological evaluation (Figure-C). she is in complete remission without treatment. 